\title{
Multi-omics analysis reveals regulators of the response to PDGF-BB treatment in pulmonary artery smooth muscle cells
}

\author{
Jidong Chen ${ }^{1,2+}$, Xiaolei Cuil ${ }^{1 \dagger}$, Zhengjiang Qian ${ }^{1,2}$, Yanjiao Li ${ }^{1,2}$, Kang Kang $^{3}$, Junle Qu ${ }^{2}, \mathrm{Li} \mathrm{Li}^{1 *}$ and Deming Gou ${ }^{1 *}$ (D)
}

\begin{abstract}
Background: Pulmonary arterial hypertension $(\mathrm{PAH})$ is a lethal disease with pronounced narrowing of pulmonary vessels due to abnormal cell proliferation. The platelet-derived growth factor BB (PDGF-BB) is well known as a potent mitogen for smooth muscle cell proliferation. To better understand how this growth factor regulates pulmonary arterial smooth muscle cells (PASMCs) proliferation, we sought to characterize the response to PDGF-BB stimulation at system-wide levels, including the transcriptome and proteome.
\end{abstract}

Results: In this study, we identified 1611 mRNAs (transcriptome), 207 proteins (proteome) differentially expressed in response to PDGF-BB stimulation in PASMCs based on RNA-sequencing and isobaric tags for relative and absolute quantification (iTRAQ) assay. Transcription factor (TF)-target network analysis revealed that PDGF-BB regulated gene expression potentially via TFs including HIF1A, JUN, EST1, ETS1, SMAD1, FOS, SP1, STAT1, LEF1 and CEBPB. Among them, SMAD1-involved BMPR2/SMADs axis plays a significant role in PAH development. Interestingly, we observed that the expression of BMPR2 was decreased in both mRNA and protein level in response to PDGF-BB. Further study revealed that BMPR2 is the direct target of miR-376b that is up-regulated upon PDGF-BB treatment. Finally, EdU incorporation assay showed that miR-376b promoted proliferation of PASMCs.

Conclusion: This integrated analysis of PDGF-BB-regulated transcriptome and proteome was performed for the first time in normal PASMCs, which revealed a crosstalk between PDGF signaling and BMPR2/SMADs axis. Further study demonstrated that PDGF-BB-induced miR-376b upregulation mediated the downregulation of BMPR2, which led to expression change of its downstream targets and promoted proliferation of PASMCs.

Keyword: Platelet-derived growth factor-BB (PDGF-BB), Pulmonary arterial hypertension (PAH), RNA sequencing, iTRAQ, BMPR2, miR-376b,

\section{Background}

Pulmonary arterial hypertension (PAH) is a life-threatening disease characterized by a sustained elevation of pulmonary arterial pressure and pulmonary vascular resistance $[1,2]$. Essential pathological characteristics of $\mathrm{PAH}$ are excessive proliferation and migration of pulmonary arterial smooth muscle cells (PASMCs), leading to medial hypertrophy and vascular remodeling $[3,4]$. PASMCs are maintained in a

\footnotetext{
* Correspondence: ylili@szu.edu.cn; dmgou@szu.edu.cn

${ }^{\dagger}$ Equal contributors

${ }^{1}$ Shenzhen Key Laboratory of Microbial Genetic Engineering, Shenzhen Key Laboratory of Marine Bioresource and Eco-environmental Science, College of Life Sciences and Oceanography, Shenzhen University, Nanhai Ave 3688, Shenzhen, Guangdong 518060, China

Full list of author information is available at the end of the article
}

quiescent and non-migratory state under normal condition. However, PASMCs proliferation and migration are significantly promoted in response to various growth factors and cytokines, such as platelet-derived growth factor-BB (PDGF-BB), fibroblast growth factor, insulin-like growth factor-1, tumor necrosis factor- $\alpha$ and interleukin-1 [5-7].

PDGF is the most potent mitogenic factor for vascular smooth muscle cells (VSMCs), and exerts its actions via binding and activating two PDGF receptor (PDGFR) subtypes, PDGFR $\alpha$ and PDGFR $\beta$ [8-10]. Abundant evidence reveals that PDGF is a major contributor to the pathobiology of vascular disorders including PAH [11, 12]. PDGF ligands are upregulated in lung tissue and pulmonary cells in monocrotaline (MCT)- and hypoxiainduced experimental PAH animal [13-16]. Similar 
alterations with upregulation of PDGF ligands and PDGFR $\beta$ were found in lung tissue and small pulmonary arteries of patients with PAH $[17,18]$. Imatinib, a PDGF receptor antagonist, has been reported to dramatically improve PAH in some human cases as well as animal models, but serious side effects and drug discontinuation are common $[19,20]$. Therefore, it is particularly necessary to investigate gene expression alteration globally in PASMCs induced by PDGF-BB.

Previously, Shirvani and his colleagues have globally determined the effect of PDGF on transcription factor CHF1/Hey2-knockout VSMCs and demonstrate that CHF1/Hey2 profoundly affects vascular smooth muscle phenotype by altering both the absolute expression level of a variety of genes and the kinetics of growth factorinduced gene expression. [21]. In another report, Lee et al. have analyzed the gene expression profile with multiple whole-genome expression array datasets and identified NFAT family members and target genes as important effectors of VSMCs in response to PDGF [22]. These studies were mainly based on traditional high throughput technology, such as microarray, which covered only portion of the whole genome [21, 22]. In addition, a recent study has integrated proteomic and transcriptomic profiles and identified a novel PDGFMYC network in bladder smooth muscle cells [23], however, this kind of analysis in PASMCs is still lacking.

To better understand how PDGF-BB regulates gene expression in PASMCs, we performed the integrated analysis of transcriptome and proteome changes in response to PDGF-BB stimulation in rat PASMCs (RPASMCs), based on next generation sequencing and relative and absolute quantification (iTRAQ) technology. Our analysis revealed a significant role of SMAD1 in PDGF-BB induced gene expression. Further study demonstrated that PDGF-BB-induced miR-376b upregulation mediated the downregulation of BMPR2, which led to expression change of SMAD1 targets and promoted proliferation of PASMCs.

\section{Results}

\section{Profiling of mRNA expression in response to PDGF-BB}

RNA deep-sequencing was carried out to identify differentially expressed genes in response to PDGF-BB. Total RNA samples for RNA-Seq analysis were obtained from RPASMCs following PDGF-BB (30 ng/ml) exposure for 0 or $12 \mathrm{~h}$ (2 independently isolated biological replicates). Every sample generated more than 90,000,000 reads after quality control, and 50,553 mRNA transcripts were identified with 373 novel transcripts that have not been reported in rat. By quantitative analysis, 1611 transcripts were revealed to express differentially in response to PDGF-BB (Q value $<0.05 ; \quad F P K M>0.5$; fold change
$(\mathrm{FC}) \geq 2$ ), with 814 up- and 797 down-regulated (Fig. 1a and Additional file 1).

Next, gene ontology (GO) analysis was performed with KOBAS 2.0 online software (http://kobas.cbi.pku.edu.cn/ ) [24]. Results showed that these differentially expressed genes were enriched significantly in GO terms involved in cell proliferation, such as cell cycle, DNA replication and cell division (Fig. 1c and Additional file 2), suggesting the significant role of PDGF-BB in smooth muscle cell proliferation as reported previously [23]. KEGG (Kyoto Encyclopedia of Genes and Genomes) pathway analysis suggested that differentially expressed genes were enriched significantly in cell cycle, DNA replication, PI3K-Akt signaling pathway, HIF-1 signaling pathway, focal adhesion. All of these pathways are functionally involved in cell proliferation and migration (Fig. 1b and Additional file 2). To find out the potential transcription factors (TFs) mediating PDGF-BB regulation on these differentially expressed mRNA, TF-targets data were collected from TRED database [25] and compared to differentially expressed mRNAs. Data analysis revealed that E2F4, HIFA, JUN, TP53, ATF, ESR1, EST1, ETS1, SMAD1 and NFKB1 were top 10 TFs in response to PDGF-BB stimulation (Fig. 1d), partly consistent with previous reports on bladder smooth muscle cells [23]. Interestingly, we identified as the first time that SMAD1, an essential TF in BMPR2/SMADs signaling pathway was regulated by PDGF-BB (Fig. 1d), implying a potential link between BMPR2/SMADs signaling and PDGF-BB stimulation.

\section{Profiling of protein expression in response to PDGF-BB}

iTRAQ-based proteomics analysis was performed in RPASMCs treated with PDGF-BB in three time points $(0,12,24 \mathrm{~h}$, duplicate per time point). Totally, 3666 proteins were identified from 26,878 peptides matched with 82,825 spectra at a FDR (false discovery rate) of $5 \%$, 2934 of which matched to two or more peptides. Among them, 207 proteins were differentially expressed in response to PDGF-BB stimulation for 12 or $24 \mathrm{~h}(p<0.05$, FC > 1.6), with 96 up- and 111 down-regulated (Fig. 2a and Additional files 3 and 4).

GO analysis suggested the differentially expressed protein were also enriched in GO terms involved in proliferation and migration, such as focal adhesion, stress fiber and wound healing (Fig. 2b). In KEGG pathway analysis, only one pathway was showed to be enriched significantly. However, among the 10 most enriched pathways, focal adhesion and regulation of actin cytoskeleton were involved tightly in migration and proliferation (Fig. 2c). TF-target analysis suggested that differentially expressed proteins majorly enriched in the TFs including MYC, HIFA, USF, JUN and REL (Fig. 2d). 


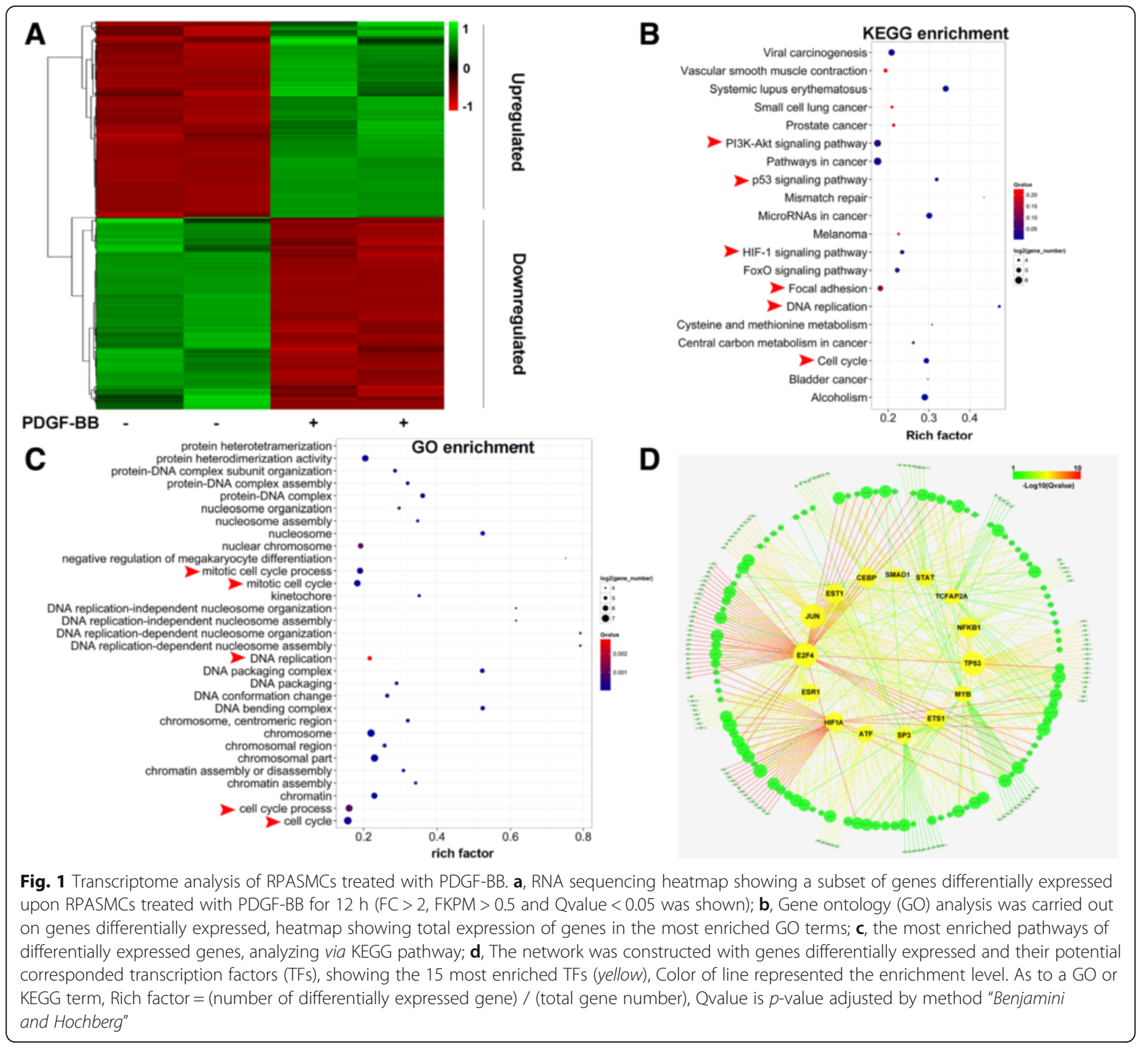

Interestingly, differentially expressed proteins were enriched significantly in SMAD1 as in mRNA level (Fig. 2d).

\section{Comparison and integration of transcriptome and proteome}

To further identify PDGF-BB mediated gene expression, the datasets from RNA-seq and ITRAQ were applied for the integrated analysis. Totally, 56 genes were found to be differentially expressed at both mRNA and protein levels (Fig. 3a). The correlation analysis suggested that these genes expressed in high correlation in the two levels $(r=0.87032, p<0.001$, Fig. $3 \mathrm{~b})$. In addition, The integrated analysis of TFs-target suggested that differentially expressed genes enriched significantly in HIF1A,
JUN, EST1, ETS1, SMAD1, FOS, SP1, STAT1, LEF1 and CEBPB, at both mRNA and proteins levels (Fig. 3c). It is well known that BMPR2/SMAD1 axis plays critical role during the PAH initiation and development $[26,27]$. Hence we chose potential targets of SMADs for further analysis. KEGG pathway analysis suggested that these differentially expressed targets enriched significantly in PI3K-Akt signaling pathway, focal adhesion and cell cycle, and all these pathways play significant roles in cell proliferation and migration (Fig. 3d).

\section{Validation of differentially expressed mRNA identified via RNA-sequencing}

Our interest focused on the proliferation, migration and differentiation of PASMCs, hence those differentially 


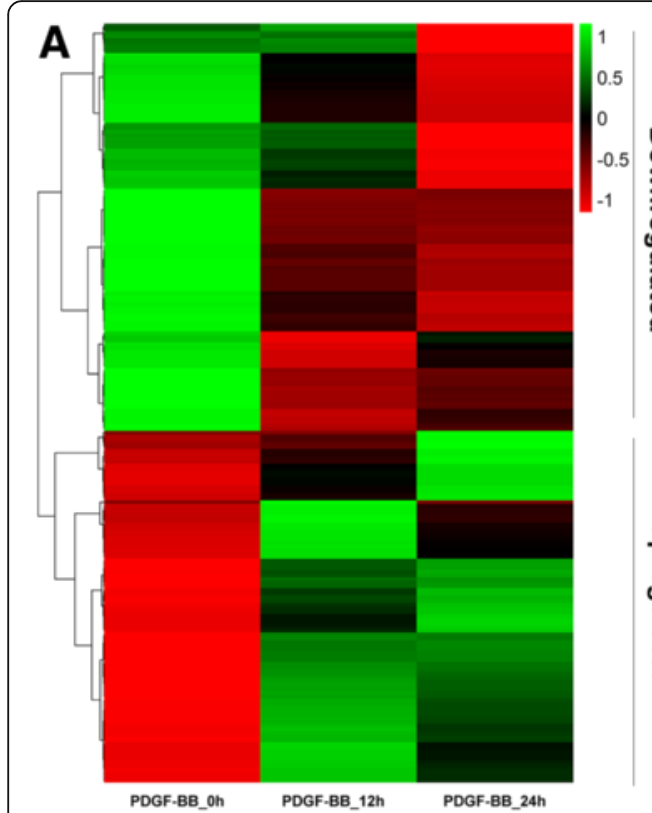

B

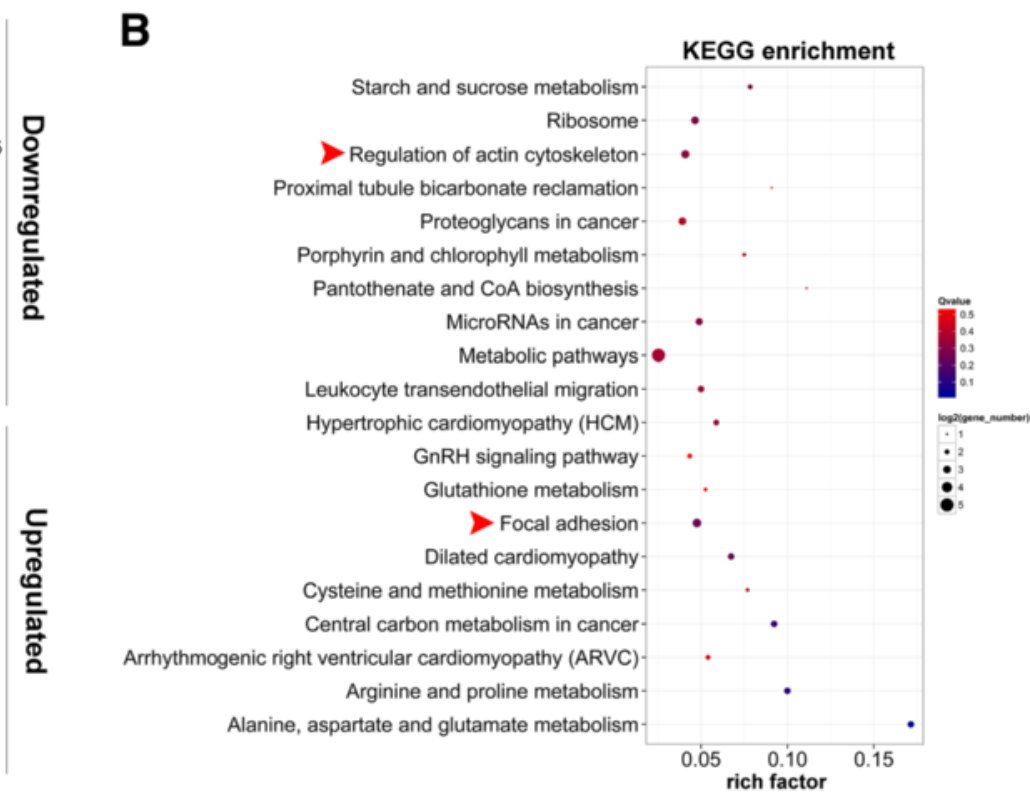

C

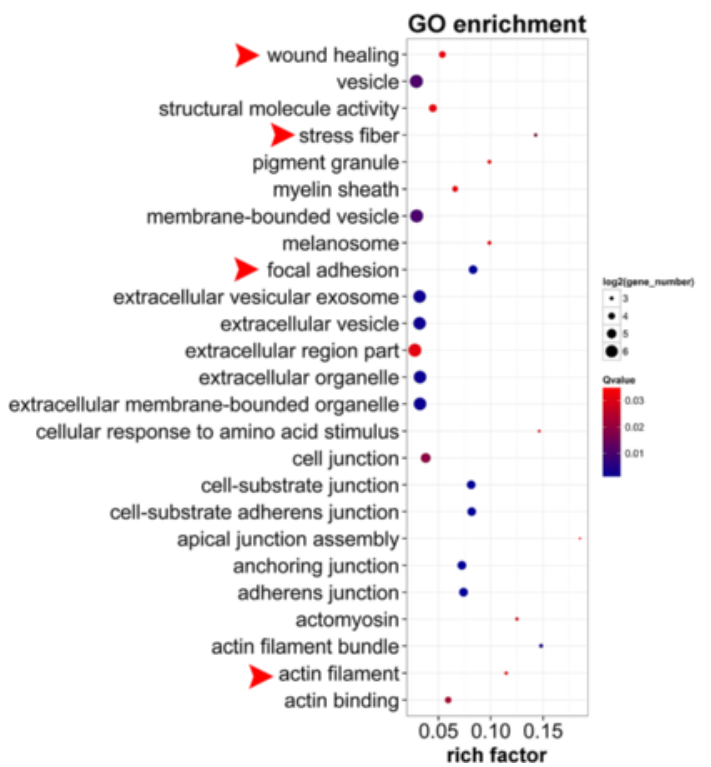

D

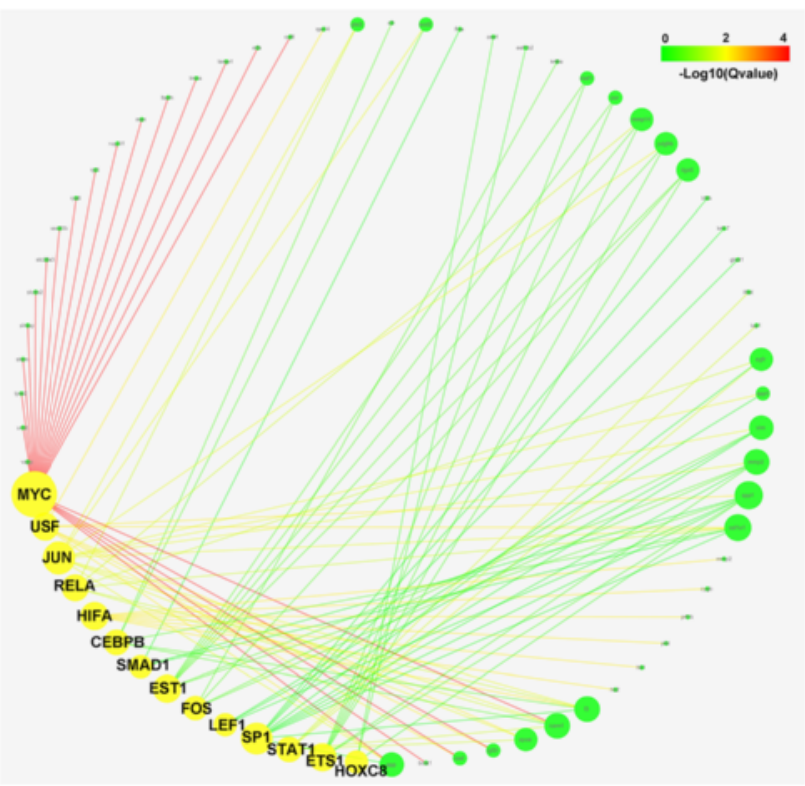

Fig. 2 Protenome analysis of RPASMCs treated with PDGF-BB. a, heatmap displaying a subset of proteins differentially expressed upon RPASMCs treated 12 or $24 \mathrm{~h}$ with PDGF-BB (FC> 1.6, $p<0.05$ ), measured via iTRAQ; $\mathbf{b}, \mathrm{GO}$ analysis showing the most enriched $\mathrm{GO}$ term on differentially expressed proteins in response to PDGF-BB; $\mathbf{c}$, the most enriched pathway of proteins differentially expressed, analyzing via KEGG pathway; $\mathbf{d}$, The network was constructed with differentially expressed proteins and their potential corresponded transcription factors (TFs), displaying the 14 significantly enriched TFs (yellow), Color of line represented the enrichment level. As to a GO or KEGG term, Rich factor = (number of differentially expressed gene) / (total gene number), Qvalue is p-value adjusted by method "Benjamini and Hochberg"

expressed targets of SMADs involved in these processes were chosen for validation via qRT-PCR (Fig. 4a). The results showed that, bambi, edn1 and $i d 2$ presented time-dependent downregulation in response to PDGF-BB treatment. On the other hand, myc, cdkn1a, jun, col2, tgfb1, gja1, col5a1 and ctnnb1 displayed time-dependent upregulation in response to PDGF-BB treatment. The results from qRT-PCR assays were consistent with RNAsequencing data, except for myc (Fig. 4b).

Taken all results above together, we revealed a significant role of SMAD1 in regulating PDGF-BB induced gene expression. Next we would try to explore how PDGF-BB impacts SMAD1 to regulate the corresponding gene expression. 


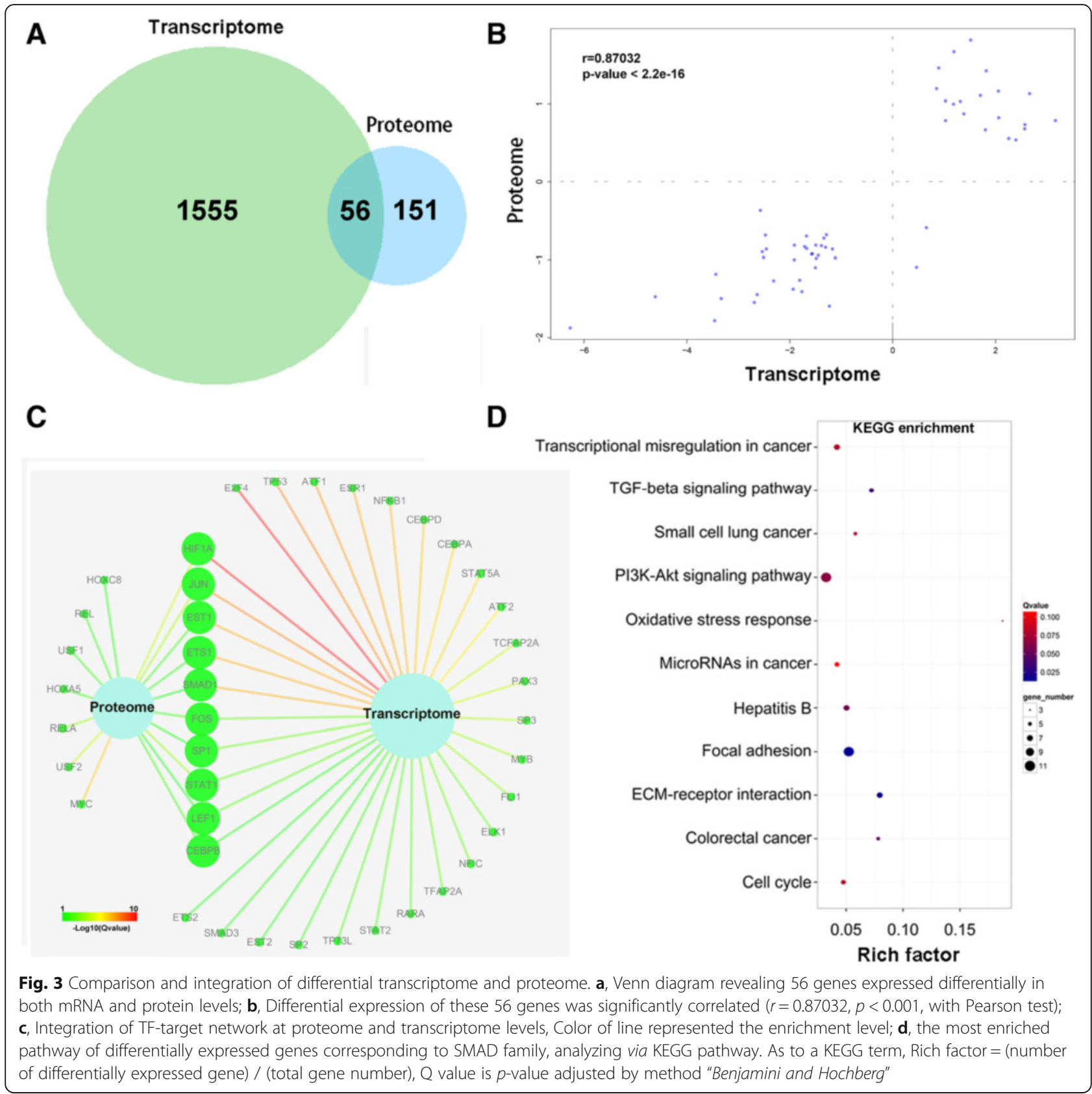

\section{Downregulation of BMPR2 led to expression alteration of SMADs targets}

To explore what accounted for SMAD1-involved gene expression in PDGF-BB stimulated RPASMCs, data from RNA-sequencing and iTRAQ assay were analyzed and BMPR2 was found to be downregulated in response to PDGF-BB treatment both in mRNA and protein levels. As well known, BMPR2 is the core upstream regulator in BMPR2/SMADs signaling. Through BMRP2-dependent BMRP1 activation, SMAD1 translocates into nucleus and interacts with other transcription factors to regulate gene expression, negatively or positively [28]. Western blot confirmed that BMPR2 protein downregulated in response to PDGF-BB with time-dependent manner in RPASMCs (Fig. 4c). qRT-PCR also validated that BMPR2 mRNA altered with time- and dose- dependent manner in response to PDGF-BB in RPASMCs (Fig. 4d, e). Taken together, theses data suggested that PDGF-BB induced BMPR2 downregulation could be causative of expression alteration of SMADs targets.

\section{miR-376b mediated the downregulation of BMPR2}

To determine if PDGF-BB regulates BMPR2 expression in post-transcriptional level, we next explored whether 


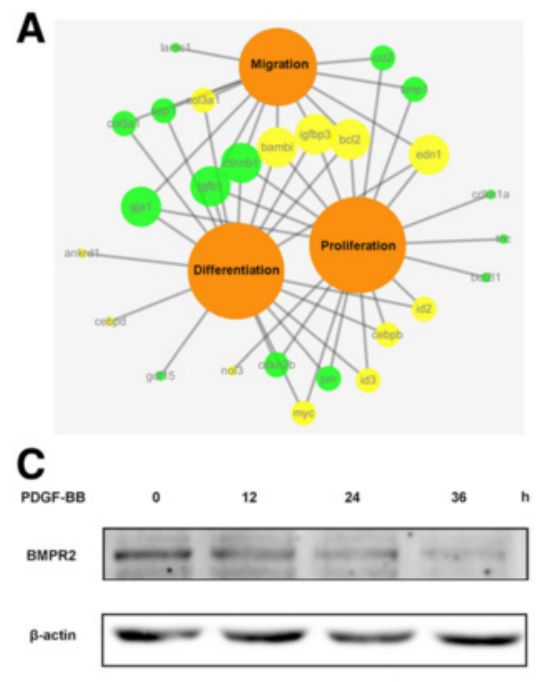

D

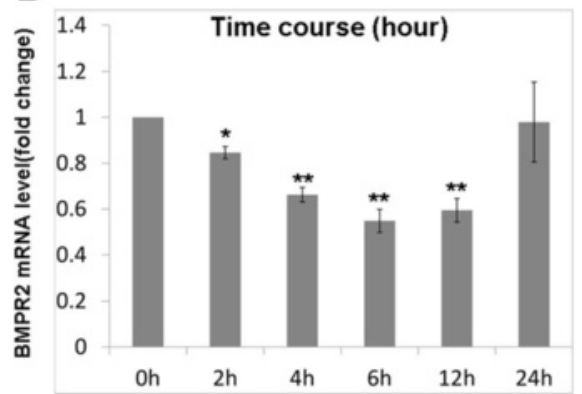

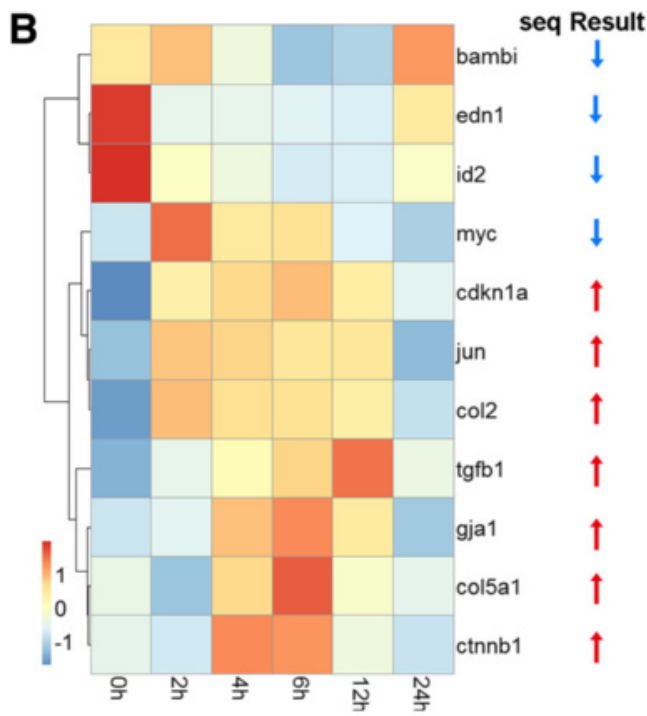

E

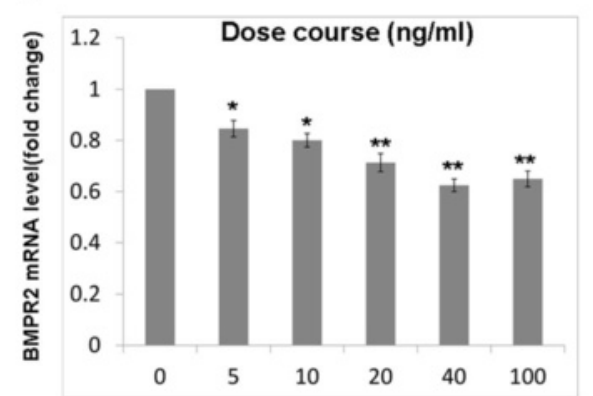

Fig. 4 Differential expression of SMAD family TFs in response to PDGF-BB was due to downregulation of BMPR2. a, differentially expressed genes corresponding to SMAD involved in 3 major cellular procedures; $\mathbf{b}$, q-PCR validated the expression of part of SMAD family targets, which were identified to express differentially in response to PDGF-BB via RNA-sequencing; BMPR2 was detected in RAPSMCs treated with PDGF-BB (time course and dose course) via Western blot at protein level $\mathbf{c}$ and qRT-PCR at mRNA level $\mathbf{d}, \mathbf{e}, n=3,{ }^{*} p<0.05,{ }^{*} p<0.01 \mathrm{vs} 0 \mathrm{~h}$ or $0 \mathrm{ng} / \mathrm{ml}$

miRNAs involved in downregulation of BMPR2. Our lab has investigated the expression profiling of $1078 \mathrm{miR}$ NAs and identified a group of miRNAs differentially expressing in HPASMCs treated by PDGF-BB [28]. Among them, 13 miRNAs were significantly changed in both human and rat PASMCs, with 7 (miR-221-5p, miR-221-3p, miR-376a, miR-376b, miR-146b, miR-7-5p, miR-210) upregulated and 6 (miR-339, miR-98, miR-107, miR-328a, miR-1281, miR-323a) downregulated in response to PDGF-BB (Additional file 5). Further study suggested that expression of these 13 miRNAs also changed with time-dependent manner following PDGFBB treatment in RPASMCs (Fig. 5a).

Using online prediction tool of Targetscan (http:// www.targetscan.org/), multiple binding sites of miR-7, miR-24 and miR-376b respectively were identified in BMPR2 3'UTR. To test whether BMPR2 is a direct target of these 3 miRNAs, the effect of miRNA mimic on BMPR2 protein level was detected firstly. Western blot showed that only miR-376b mimic could inhibit BMPR2 protein level (Fig. 5b). In addition, HEK-293 cells that co-transfected with miR-376b mimic and a luciferase construct containing sequence of BMPR2 3'UTR resulted in significant decrease in luciferase activity (Fig. 5d), but not miR-7 and miR-24 (data not showed). Two potential miR-376b binding sites within BMPR2 3'UTR have been identified via Targetscan (Fig. 5c), however, they exhibited great difference in their susceptibility to miR-376b mediated repression. Mutation on the first binding site (Mut1) completely abolished the inhibitory effect caused by miR-376b, but not the second binding site mutated (Mut2) (Fig. 5d). Together, these data demonstrated that upregulation of miR-376b in response to PDGF-BB repressed the BMPR2 protein level post-transcriptionally.

\section{miR-376b promoted proliferation of RPASMCs}

A series of reports have suggested that downregulation of BMPR2 promoted proliferation of PASMCs [4]. Therefore, we investigated whether miR-376b could impact proliferation of RPASMCs. EdU incorporation assay showed that, miR-376b mimic significantly promoted 


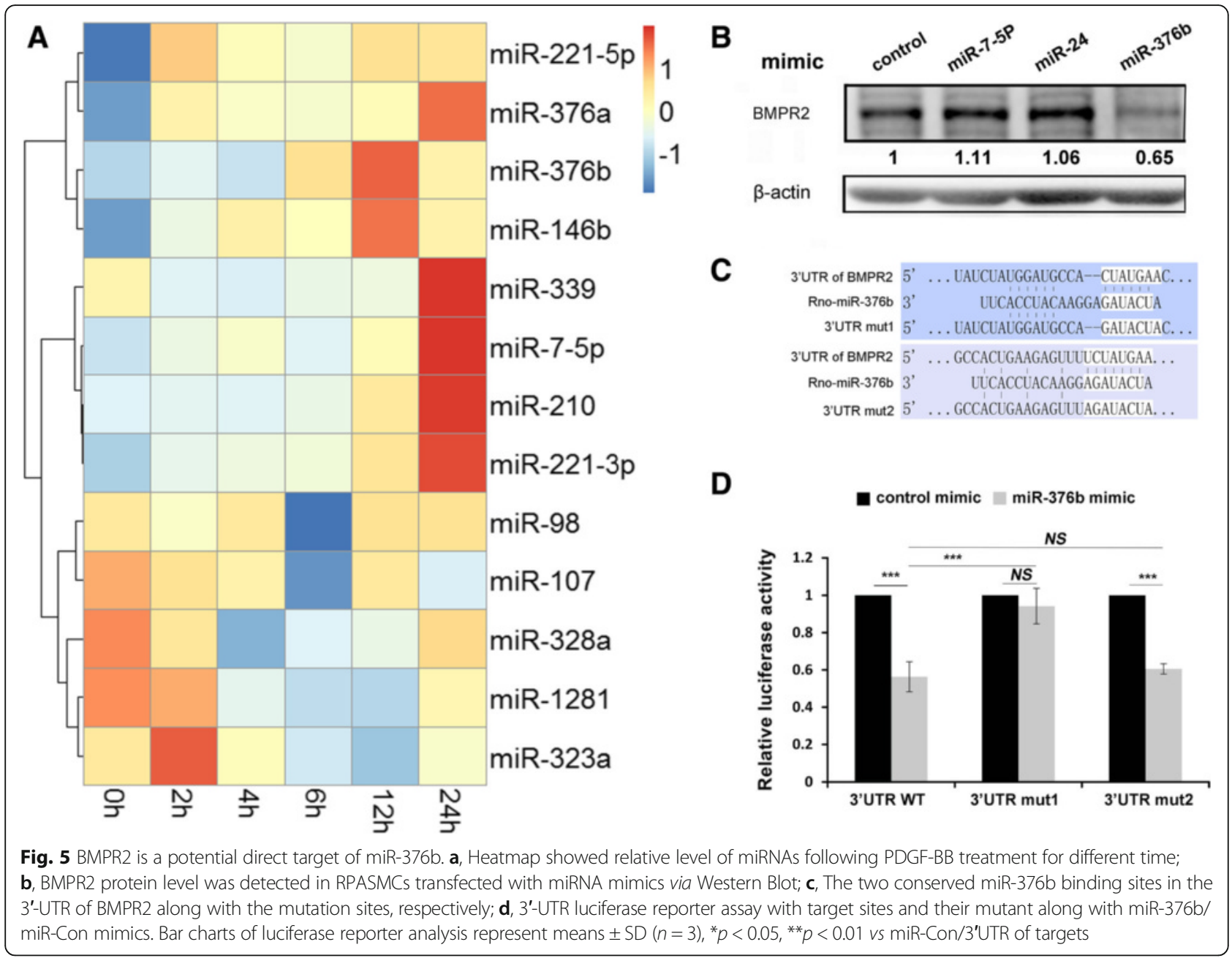

proliferation of RPASMCs (Fig. 6a, b). This data further demonstrated that miR-376b could inhibit BMPR2 expression and promote proliferation of RPASMCs.

In summary, this study integrated transcriptome and proteome and discovered that, in response to PDGF-BB, miR-376b was upregulated to repress BMPR2 level. Decreased BMPR2 further impact downstream SMADs and altered target genes expression, which promoted proliferation of RPASMCs (Fig. 6d).

\section{Discussion}

PDGF signaling plays important role in pulmonary vascular remodeling, however, the detail mechanism underlying this process remains elusive. There has been a set of genome-wide studies about PDGF-responsive gene expression in VSMCs, which mainly based on traditional high throughput technology, such as microarray, covering only portion of the whole transcriptome [21, 22]. Moreover, the four fundamental cellular processes, including transcription, mRNA degradation, translation and protein degradation, make the correlation between mRNA and protein more complicated. Therefore, integrated analysis of mRNA and protein levels would provide more comprehensive information to understand gene regulation [29]. However, integrating transcriptome and proteome in VSMCs is still lacking [23]. In this study, we utilized the next generation sequencing, combining iTRAQ isobaric labeling technology to globally study the gene expression regulation in PASMCs in response to PDGF-BB. Our results confirmed the crucial role of transcription factors JUN and MYC in PASMCs proliferation and migration, as previously suggested [23]. Moreover, our integrated analysis extended the understanding of PDGF-BB function on PASMCs, revealing the crosstalk between PDGF signaling and BMPR2/SMADs axis mediated by miR-376b. Previous report suggested that BMP/ BMPR2/SMADs signaling prevented PDGF-BB-induced proliferation of PASMCs by decreasing nuclear phosphoERK via its transcriptional target apoE [30]. Our work suggested that PDGF signaling could regulate BMPR2/ 


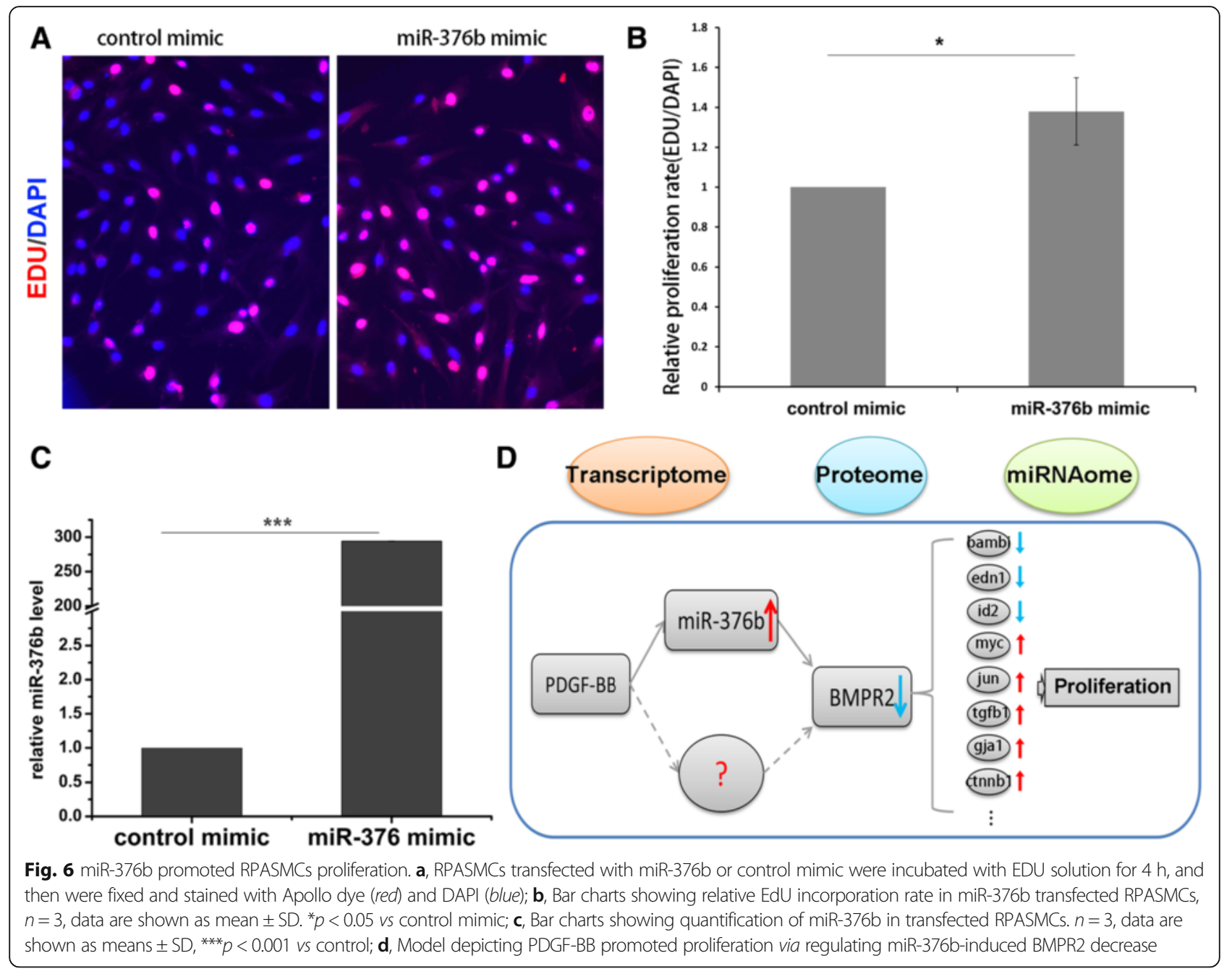

SMADs signaling and hence promote the proliferation of PASMC in the other hand.

Integrated analysis indicated that PDGF-BB impacted targets expression of 10 transcription factors, namely HIF1A, JUN, EST1, ETS1, SMAD1, FOS, SP1, STAT1, LEF1 and CEBPB (Fig. 3c), both in mRNA and protein level. Mutations or downregulation of BMPR2 is presented in most cases of heritable $\mathrm{PAH}(\mathrm{HPAH})$, indicating a functional role of BMPR2 in PAH initiation/progression [4]. Hence we focused on exploring the effect of PDGF$\mathrm{BB}$ on BMP/BMPR2/SMADs signaling. In this study, we identified 11 alternatively expressed genes that are potential targets of BMP/BMPR2/SMADs axis. Among them bambi, edn1 and $i d 2$ were downregulated in a timedependent manner, while myc, cdkn1a, jun, col2, tgfb1, gja1, col5a1 and ctnnb1 were upregulated (Fig. 4b). These genes have been annotated with important roles in mediating the proliferation, migration and differentiation of PASMCs [23, 31-38], and our finding further suggested their cooperation in mediating the PDGF-induced dysfunction of PASMCs. For instance, since bambi negatively regulates TGF- $\beta$-family signaling [31], bambi downregulation and $\operatorname{tg} f b 1$ upregulation indicated that PDGF signaling could positively regulate TGF- $\beta$ signaling. Previous reports showed that TGF- $\beta$ signaling inhibits PASMCs proliferation in normal condition but promotes proliferation in BMPR2-mutated PASMCs [39]. Therefore, activated PDGF signaling in PASMCs would lead to increased TGF- $\beta$ signaling and reduced BMP signaling, both of which are implicated in PAH pathogenesis. HIF1A is another TF deserved paying attention to, since many reports have suggested the important role of HIF1A in pulmonary vascular remodeling [26, 27]. Our analysis revealed that, differentially expressed genes in response to PDGF-BB were enriched the most significantly in HIF1A, in both mRNA and protein levels (Fig. 3c). Hence, our data indicated the tight connection between PDGF signaling and hypoxia, which has received little attention [40].

Few researches have focused on the regulation of BMPR2 expression in PASMCs. A report suggested that miR-21 potentially suppressed BMPR2 protein level [41]. MicroRNA (miRNA) is a group of small endogenous 
noncoding single-strand RNAs ( 21-25 nt), which negatively regulate gene expression in translation phase [42]. miRNA has been emerged as key player in cardiovascular diseases and cancer development and progression and, more recently, in PAH pathogenesis [42]. Several groups has globally explored the differentially expressed miRNAs in response to PDGF-BB in PASMCs. For example, Brandi N. D and his colleagues cloned and sequenced miRNAs expressed in PASMCs under vehicle- or PDGF-BB-treated conditions and found that miR-221 was one of the few miRNAs enriched in PDGFBB-treated PASMCs, which served as a modulator of the phenotypic change of PASMCs via targeting c-Kit and p27Kip1 [43]. Li et al. colleagues performed miRNA microarray analysis in human aortic smooth muscle cells (SMCs) stimulated with PDGF-BB and identified miR638 as one of the most significantly downregulated miRNA in human VSMCs in response to PDGF-BB stimulation [7]. Together, these data highlighted the contribution of the miRNA regulation in PDGF signaling pathway. Our lab utilized an improved miRNA detection assay, S-Poly (T) plus assay, to profile the expression of 987 miRNAs and found 13 miRNAs with altered expression in HPASMCs after PDGF-BB stimulation. To investigate whether miRNAs involved in PDGF-BB induced BMPR2 downregulation, online software Targetscan were used to predict the binding sites of those 13 miRNAs, 3 of which possessed the potential binding sites in the 3'UTR of BMPR2. However, Western blot showed that only miR-376b mimic could inhibit the expression of BMPR2 protein.

Our data suggested that PDGF-BB treatment induced downregulation of BMPR2 not only at protein level but also at mRNA level. miR-376b could inhibit the expression of BMPR2 at protein level (Fig. 5b), however, BMPR2 mRNA in PASMCs remained unchanged after miR-376b mimic transfection (data not shown), suggesting the inhibitory effect of miR-376b on BMPR2 is posttranscriptional. As to the transcriptional regulation of BMPR2 expression, previous study showed that BMPR2 transcription could be affected by methylation and acetylation in endothelial cells [44]. However, it appeared not the case in PASMC upon PDGF stimulation, as suppressed methylation with 5-Azacytidine and deacetylation with Trichostatin A failed to recover the BMPR2 mRNA levels inhibited by PDGF-BB (data not shown). Therefore, we hypothesize that PDGF-BB regulates BMPR2 transcription through impacting promoter activity and further investigation is needed to verify this hypothesis.

\section{Conclusion}

In conclusion, our results provided the first systems-level integrated analysis of PDGF-BB-regulated transcriptome, proteome and miRNAome (3-omics) in PASMCs. The results demonstrated that PDGF-BB-induced miR-376b upregulation mediated BMPR2 downregulation, which led to expression change of its target genes and promoted proliferation of PASMCs.

\section{Method \\ Ethics statement}

All experiments were carried out according to China Council on Animal Care and the protocols used were approved by the Animal Care and Use Committee of Guangdong Province, China.

\section{Cell culture and transfection}

RPASMCs were generous gifts from Dr. Zeng Yan, and cultured as previously reported [45]. Cultured primary RPASMCs ( $\leq 4$ passage) were starved for $12 \mathrm{~h}(\mathrm{~h})$ and then treated with PDGF-BB ( $30 \mathrm{ng} / \mathrm{ml}, \mathrm{R} \& D$ system, Minneapolis, MN) for different time $(0,2,4,6,12,24 \mathrm{~h})$. miRNA mimics $(20 \mathrm{nM})$ were transfected with $\mathrm{K} 2$ transfection reagent (Biontex, Planegg, Germany) in RPASMCs. HEK293a, a cell line derived from human embryonic kidney, was purchased from American Type Culture Collection (Manassas, USA). The transfection of miRNA mimic and DNA plasmids into HEK-293 cells were performed with polyethylenimine (PEI, Geneups, Shenzhen, China).

\section{RNA deep-sequencing and identification of differentially expressed mRNA}

Total RNAs were extracted with RNAiso Plus (Takara biotechnology Co., Dalian, China). Following procedure was performed by the sequencing company (Novogene Bioinformatics Technology Co., Beijing, China). Briefly, Sequencing libraries were generated using the rRNAdepleted RNAbyNEBNext ${ }^{\circ}$ Ultra $^{\mathrm{Tu}}$ Directional RNA Library Prep Kit for Illumina ${ }^{\circ}$ (NEB, USA) following manufacturer's recommendations. The libraries were sequenced on an Illumina Hiseq 2000 platform and $100 \mathrm{bp}$ paired-end reads were generated. Demultiplexed and quality filtered reads were then aligned to Rat reference assembly (Rn5) using TopHat (v.2.0.9) [46]. The mapped reads of each sample were assembled by both Scripture (beta2) [47] and Cufflinks (v2.1.1) [48] in a reference-based approach.

Cuffdiff (v2.1.1) was used to calculate FPKMs (fragments per kilo-base of exon per million fragments mapped) of coding genes in each sample [48]. Gene FPKMs were computed by summing the FPKMs of transcripts in each gene group. Cuffdiff provides statistical routines for determining differential expression in digital transcript or gene expression data using a model based on the negative binomial distribution [48]. Genes with a $\mathrm{Q}$ value $<0.05$, FKPRM $>0.5$ and $\mathrm{FC}>2$ were assigned as differentially expressed. 


\section{GO and KEGG analysis}

GO-term analysis was utilized to determine the potential functions of differentially expressed genes in response to PDGF-BB. GO term with Q-values $\leq 0.05$ was considered to be significantly enriched. Meanwhile, KEGG analysis was used to assess the pathway that differential genes may involve in. KEGG term with Q-values $\leq 0.05$ was considered to be significantly enriched. Both GO and KEGG analysis were performed with KOBAS 2.0 online software (http://kobas.cbi.pku.edu.cn/) [24].

\section{Identification of key transcription factors (TFs) regulating differentially expressed genes}

To identify key TFs, TF-target interaction data for 179 TFs were collected from public database TRED to build a expanded list of rat TF-target interaction, which includes homologous human and mouse ones [25]. We utilized the enrichment level to valuate the significance of TFs in PDGF-BB regulating gene expression, which was assessed with Fisher's exact test, and the FDR correction method is "Benjamini and Hochberg".

\section{iTRAQ labeling and Identification of differentially expressed proteins}

ITRAQ labeling was performed using the 8-plex iTRAQ reagents (Applied Biosystems, Foster City, CA, USA) as previously reported [49]. Then, the labeled samples were lyophilized and assigned to HPLC-MS/MS (high performance liquid chromatography-mass spectrometry/ mass spectrometry) analysis in HPLC electrospray ionization MS/MS system (Applied Biosystems, Foster City, CA, USA).

Quality assessment of the iTRAQ datasets was performed as described [49]. The statistical differences between two groups were assessed with the double-sided Student's $t$ test. Additionally, fold-change $=$ average $(12$ or $24 \mathrm{~h}) /$ average $(0 \mathrm{~h})$. The differentially expressed proteins were identified using the following criteria: 1 ) overall $P$ values are less than $0.05 ; 2$ ) proteins quantified in at least two replicates; and 3) absolute fold changes are larger than 1.6.

\section{Quantitative RT-PCR}

For mRNA detection, total RNA was reversely transcribed using M-MLV Reverse Transcriptase (TaKaRa, Dalian, China) with oligo $(\mathrm{dT})_{18}$ plus random hexamer primers (Promega, Madison, WI). qRT-PCR was performed with gene specific primers and SYBR Green PCR Master Mix (Applied Biosystems, Foster City, CA) on ABI StepOne real-time PCR System (Applied Biosystems, Foster City, CA, USA). The expression level of each gene was normalized to internal control $\beta$-actin gene and the expression level of each mRNA was calculated using the $2^{(-\Delta \Delta C T)}$ method. The S-Poly $(T)$
Plus method was used for miRNA detection as previously reported [50]. The snoRNA-202 was used as an internal control. Primers used for reverse transcription and qRT-PCR were summarized in Additional file 6.

\section{Western blot}

Cells were lysed with ice-cold RIPA ( $50 \mathrm{mM}$ Tris- $\mathrm{HCl}$, $\mathrm{pH}$ 7.5; $150 \mathrm{mM} \mathrm{NaCl} ; 1$ \% NP-40; 0.25\%sodium deoxycholate, $1 \mathrm{mM}$ EDTA) buffer supplemented with protease inhibitor cocktail (Sigma-Aldrich Inc., St. Louis, $\mathrm{MO})$. Each sample with $30 \mu \mathrm{g}$ protein were then electrophoresed on the sodium dodecyl sulfate (SDS) polyacrylamide gel and then electroblotted to nitrocellulose filter membranes (Millipore, Bedford, MA). Membranes were immersed in blocking buffer ( $5 \%$ degreased milk powder) for $1 \mathrm{~h}$ and incubated with antibodies against BMPR2 (ProteinTech Group, Chicago, IL), $\beta$-actin (SantaCruz Biotechnology, Santa Cruz, CA) overnight at $4{ }^{\circ} \mathrm{C}$. Next, the membranes were washed and incubated with horseradish peroxidase-conjugated secondary antibodies (Jackson Immuno-Research, West Grove, PA) for $1 \mathrm{~h}$ at room temperature. The protein bands were visualized with the SuperSignal chemiluminescent detection module (Pierce, Thermo Scientific Inc., San Jose, CA).

\section{3'UTR luciferase reporter assay}

TargetScan algorithm (http://www.targetscan.org) was applied to predict targets and the miRNA binding sites. The 3'UTR of BMPR2 were PCR amplified and inserted into pGL4-plasmid (Promega). The corresponding mutant constructs with six mutated residues in the region of seeding sequence were generated by site-directed mutagenesis. Luciferase activity was measured in cell extracts with a Lumat LB9508 luminometer (Berthold, Bad Wildbad, Germany). The primers used were as follow: cloning $3^{\prime}$ UTR of BMPR2: 5- GGA ATT CCC CGC CTT GTT ATC AGT CG-3 (forward) and 5- CCG CTC GAG TTA CAG CAA GCC TTT TAA ACC T -3 (reverse); BMPR2-mut1, 5-GAT GCC AGA TAC TAC GCT GAC ATT AAG CCA CTG A-3 (forward) and 5-TCA GCG TAG TAT CTG GCA TCC ATA GAT AAT ACA AAA G-3 (reverse); BMPR2-mut2, 5- GAA GAG TTT AGA TAC TAT AAG TGT AAG TAA ATG CTT TG-3 (forward) and 5- TAC ACT TAT AGT ATC TAA ACT CTT CAG TGG CTT AAT G -3 (reverse).

\section{Proliferation measurement (EdU incorporation assay)}

EdU labeling was performed using the EdU Assay Kit (Ribobio, Guangzhou, China) as recommended by the manufacturer. Briefly, approximately $1 \times 10^{4}$ cells were seeded intriplicate in 48-well plates, and the cells were cultured for $24 \mathrm{~h}$ and then transfected with miR-376b or control mimic for $48 \mathrm{~h}$, then exposed to $20 \mu \mathrm{M}$ EdU for $4 \mathrm{~h}$ at $37{ }^{\circ} \mathrm{C}$. The cells were then fixed in $4 \%$ 
paraformaldehyde for $30 \mathrm{~min}$ at room temperature and permeabilized in $0.5 \%$ Triton X-100 for $10 \mathrm{~min}$. Cells were washed with PBS, and each well was incubated with $200 \mu \mathrm{l} 1 \times$ Apollo reaction cocktail for $30 \mathrm{~min}$. DNA was then stained with $1 \mu \mathrm{g} / \mathrm{ml}$ DAPI $(200 \mu \mathrm{l}$ per well) for $30 \mathrm{~min}$ and imaged under a fluorescent microscope. All data are shown as a percentage of control mimic.

\section{Statistical analysis}

Except for those omics data, all data shown are mean values of at least three experiments with standard deviation (SD). Correlation test was performed with Pearson method. When only two groups were compared, the statistical differences were assessed with the doublesided Student's $t$ test. Significant differences between groups were analyzed using one-way ANOVA. A $p$ value less than 0.05 was considered significant.

\section{Additional files}

Additional file 1: Table S1. Differentially expressed mRNA in RPASMCs treated with PDGF-BB for 12 h. (XLSX $185 \mathrm{~kb}$ )

Additional file 2: $G O$ and $K E G G$ analysis for differentially expressed mRNA. (XLSX $956 \mathrm{~kb}$ )

Additional file 3: Table S2. Differentially expressed protein in RPASMCs treated with PDGF-BB for $12 \mathrm{~h}$. (XLSX $33 \mathrm{~kb}$ )

Additional file 4: Table S3. Table S2. Differentially expressed protein in RPASMCs treated with PDGF-BB for $24 \mathrm{~h}$. (XLSX $42 \mathrm{~kb}$ )

Additional file 5: Differentially expressed miRNA in HPASMCs. (XLSX $9 \mathrm{~kb}$ )

Additional file 6: Primers for qRT-PCR. (XLSX $15 \mathrm{~kb})$

\section{Abbreviations}

FC: Fold change; FDR: False discovery rate; FPKMs: Fragments per kilo-base of exon per million fragments mapped; GO: Gene ontology; HPAH: Heritable PAH; HPASMCs: Human PASMCs; HPLC-MS/MS: High performance liquid chromatography-mass spectrometry/ mass spectrometry; iTRAQ: Relative and absolute quantification; KEGG: Kyoto Encyclopedia of Genes and Genomes; MCT: Monocrotaline; PAH: Pulmonary arterial hypertension; PASMCs: Pulmonary arterial smooth muscle cells; PDGF-BB: Platelet-derived growth factor BB; PDGFR: PDGF receptor; qRT-PCR: Quantificational Real-time Polymerase Chain Reaction; RPASMCs: Rat PASMCs; SD: Standard deviation; TF: Transcription factor; UTR: Untranslated regions; VSMCs: Vascular smooth muscle cells

\section{Funding}

This study was partly supported by National Natural Science Foundation of China [81170047, 81370151 and 81570046 to D.G., 31571199 to K.K., and 81500044 to Z.Q.]; Shenzhen Municipal Basic Research Program [JC201006010725A to D.G.]; Interdisciplinary Innovation Team Project of Shenzhen University; Shenzhen Overseas High-Level Talents Innovation Program [YFZZ20111009 to D.G.]; Shenzhen High-tech Development Project [CXZZ20140828163951592 to D.G.]

\section{Availability of data and materials}

The RNA sequencing data will be submitted to SRA (SRX1715113). The mass spectrometry proteomics data will be deposited to the ProteomeXchange Consortium via the PRIDE partner repository. Accession number will be supplied later.

\section{Authors' contributions}

DG, LL and JC conceived and planned the study. JC and ZQ collected and extracted total RNAs for next-generation sequencing. JC and JQ analyzed the sequencing data. JC, XC, YL and KK carried out the experimental work. JC and DG wrote the manuscript. All authors read and approved the final manuscript.

\section{Competing interests}

The authors declare that they have no competing interests.

\section{Author details}

1'Shenzhen Key Laboratory of Microbial Genetic Engineering, Shenzhen Key Laboratory of Marine Bioresource and Eco-environmental Science, College of Life Sciences and Oceanography, Shenzhen University, Nanhai Ave 3688, Shenzhen, Guangdong 518060, China. ${ }^{2}$ Key Laboratory of Optoelectronic Devices and Systems of Ministry of Education and Guangdong Province, College of Optoelectronic Engineering Shenzhen University, Shenzhen, Guangdong 518060, China. ${ }^{3}$ Department of Physiology, Shenzhen University Health Science Center, Shenzhen, Guangdong 518060, China.

Received: 23 April 2016 Accepted: 26 September 2016

Published online: 06 October 2016

\section{References}

1. Lai YC, Potoka KC, Champion HC, Mora AL, Gladwin MT. Pulmonary arterial hypertension: the clinical syndrome. Circ Res. 2014;115(1):115-30.

2. Montani D, Gunther S, Dorfmuller P, Perros F, Girerd B, Garcia G, Jais X, Savale L, Artaud-Macari E, Price LC, et al. Pulmonary arterial hypertension. Orphanet J Rare Dis. 2013:8:97.

3. Morrell NW, Adnot S, Archer SL, Dupuis J, Jones PL, MacLean MR, McMurtry IF, Stenmark KR, Thistlethwaite PA, Weissmann N, et al. Cellular and molecular basis of pulmonary arterial hypertension. J Am Coll Cardiol. 2009;54(1 Suppl):S20-31.

4. Soubrier F, Chung WK, Machado R, Grunig E, Aldred M, Geraci M, Loyd JE, Elliott CG, Trembath RC, Newman JH, et al. Genetics and genomics of pulmonary arterial hypertension. J Am Coll Cardiol. 2013;62(25 Suppl):D13-21.

5. Millette E, Rauch BH, Kenagy RD, Daum G, Clowes AW. Platelet-derived growth factor-BB transactivates the fibroblast growth factor receptor to induce proliferation in human smooth muscle cells. Trends Cardiovasc Med. 2006;16(1):25-8.

6. Bayes-Genis A, Conover CA, Schwartz RS. The insulin-like growth factor axis: A review of atherosclerosis and restenosis. Circ Res. 2000;86(2):125-30.

7. Li P, Liu Y, Yi B, Wang G, You X, Zhao X, Summer R, Qin Y, Sun J. MicroRNA638 is highly expressed in human vascular smooth muscle cells and inhibits PDGF-BB-induced cell proliferation and migration through targeting orphan nuclear receptor NOR1. Cardiovasc Res. 2013;99(1):185-93.

8. Heldin $\mathrm{CH}$, Westermark B. Mechanism of action and in vivo role of plateletderived growth factor. Physiol Rev. 1999:79(4):1283-316.

9. Kazlauskas A. Receptor tyrosine kinases and their targets. Curr Opin Genet Dev. 1994:4(1):5-14.

10. Rosenkranz S, Kazlauskas A. Evidence for distinct signaling properties and biological responses induced by the PDGF receptor alpha and beta subtypes. Growth Factors. 1999;16(3):201-16.

11. Raines EW. PDGF and cardiovascular disease. Cytokine Growth Factor Rev. 2004;15(4):237-54.

12. Grimminger F, Schermuly RT. PDGF receptor and its antagonists: role in treatment of PAH. Adv Exp Med Biol. 2010;661:435-46.

13. Arcot SS, Lipke DW, Gillespie MN, Olson JW. Alterations of growth factor transcripts in rat lungs during development of monocrotaline-induced pulmonary hypertension. Biochem Pharmacol. 1993;46(6):1086-91.

14. Cai Y, Han M, Luo L, Song W, Zhou X. Increased expression of PDGF and c-myc genes in lungs and pulmonary arteries of pulmonary hypertensive rats induced by hypoxia. Chin Med Sci J. 1996:11(3):152-6.

15. Kwapiszewska G, Wilhelm J, Wolff S, Laumanns I, Koenig IR, Ziegler A, Seeger W, Bohle RM, Weissmann N, Fink L. Expression profiling of laser-microdissected intrapulmonary arteries in hypoxia-induced pulmonary hypertension. Respir Res. 2005:6:109.

16. Jones R, Capen D, Jacobson M, Munn L. PDGF and microvessel wall remodeling in adult rat lung: imaging PDGF-AA and PDGF-Ralpha molecules in progenitor smooth muscle cells developing in experimental pulmonary hypertension. Cell Tissue Res. 2006;326(3):759-69.

17. Humbert M, Monti G, Fartoukh M, Magnan A, Brenot F, Rain B, Capron F, Galanaud P, Duroux P, Simonneau G, et al. Platelet-derived growth factor expression in primary pulmonary hypertension: comparison of HIV seropositive and HIV seronegative patients. Eur Respir J. 1998;11(3):554-9. 
18. Lanner MC, Raper M, Pratt WM, Rhoades RA. Heterotrimeric G proteins and the platelet-derived growth factor receptor-beta contribute to hypoxic proliferation of smooth muscle cells. Am J Respir Cell Mol Biol. 2005;33(4):412-9.

19. Berghausen $\mathrm{E}$, ten Freyhaus $\mathrm{H}$, Rosenkranz S. Targeting of platelet-derived growth factor signaling in pulmonary arterial hypertension. Handb Exp Pharmacol. 2013:218:381-408.

20. Gomberg-Maitland M, Bull TM, Saggar R, Barst RJ, Elgazayerly A, Fleming TR, Grimminger F, Rainisio M, Stewart DJ, Stockbridge N, et al. New trial designs and potential therapies for pulmonary artery hypertension. J Am Coll Cardiol. 2013;62(25 Suppl):D82-91.

21. Shirvani SM, Mookanamparambil L, Ramoni MF, Chin MT. Transcription factor CHF1/Hey2 regulates the global transcriptional response to platelet-derived growth factor in vascular smooth muscle cells. Physiol Genomics. 2007;30(1):61-8.

22. Lee MY, Garvey SM, Baras AS, Lemmon JA, Gomez MF, Schoppee Bortz PD, Daum G, LeBoeuf RC, Wamhoff BR. Integrative genomics identifies DSCR1 (RCAN1) as a novel NFAT-dependent mediator of phenotypic modulation in vascular smooth muscle cells. Hum Mol Genet. 2010;19(3):468-79.

23. Yang W, Ramachandran A, You S, Jeong H, Morley S, Mulone MD, Logvinenko T, Kim J, Hwang D, Freeman MR, et al. Integration of proteomic and transcriptomic profiles identifies a novel PDGF-MYC network in human smooth muscle cells. Cell Commun Signal. 2014;12:44.

24. Xie C, Mao X, Huang J, Ding Y, Wu J, Dong S, Kong L, Gao G, Li CY, Wei L. KOBAS 2.0: a web server for annotation and identification of enriched pathways and diseases. Nucleic Acids Res. 2011;39(Web Server issue):W316-322.

25. Zhao F, Xuan Z, Liu L, Zhang MQ. TRED: a Transcriptional Regulatory Element Database and a platform for in silico gene regulation studies. Nucleic Acids Res. 2005;33(Database issue):D103-107.

26. Robinson JC, Graham BB, Rouault TC, Tuder RM. The crossroads of iron with hypoxia and cellular metabolism. Implications in the pathobiology of pulmonary hypertension. Am J Respir Cell Mol Biol. 2014;51(6):721-9.

27. Tuder RM, Archer SL, Dorfmuller P, Erzurum SC, Guignabert C, Michelakis E, Rabinovitch M, Schermuly R, Stenmark KR, Morrell NW. Relevant issues in the pathology and pathobiology of pulmonary hypertension. J Am Coll Cardiol. 2013;62(25 Suppl):D4-12.

28. Qian Z, Zhang L, Chen J, Li Y, Kang K, Qu J, Wang Z, Zhai Y, Li L, Gou D. MiR328 targeting PIM-1 inhibits proliferation and migration of pulmonary arterial smooth muscle cells in PDGFBB signaling pathway. Oncotarget. 2016. doi:10. 18632/oncotarget.10714. [Epub ahead of print]

29. Schwanhausser B, Busse D, Li N, Dittmar G, Schuchhardt J, Wolf J, Chen W, Selbach M. Global quantification of mammalian gene expression control. Nature. 2011:473(7347):337-42.

30. Hansmann G, de Jesus Perez VA, Alastalo TP, Alvira CM, Guignabert C, Bekker JM, Schellong S, Urashima T, Wang L, Morrell NW, et al. An antiproliferative BMP-2/PPARgamma/apoE axis in human and murine SMCs and its role in pulmonary hypertension. J Clin Invest. 2008;118(5):1846-57.

31. Onichtchouk D, Chen YG, Dosch R, Gawantka V, Delius H, Massague J, Niehrs C. Silencing of TGF-beta signalling by the pseudoreceptor BAMBI. Nature. 1999:401(6752):480-5.

32. Yamanaka R, Otsuka F, Nakamura K, Yamashita M, Otani H, Takeda M, Matsumoto $\mathrm{Y}$, Kusano KF, Ito $\mathrm{H}$, Makino $\mathrm{H}$. Involvement of the bone morphogenetic protein system in endothelin- and aldosterone-induced cell proliferation of pulmonary arterial smooth muscle cells isolated from human patients with pulmonary arterial hypertension. Hypertens Res. 2010;33(5):435-45

33. Pellegrino S, Ferri N, Colombo N, Cremona E, Corsini A, Fanelli R, Gelmi ML, Cabrele C. Synthetic peptides containing a conserved sequence motif of the Id protein family modulate vascular smooth muscle cell phenotype. Bioorg Med Chem Lett. 2009;19(22):6298-302.

34. Li J, Li P, Zhang Y, Li GB, Zhou YG, Yang K, Dai SS. c-Ski inhibits the proliferation of vascular smooth muscle cells via suppressing Smad3 signaling but stimulating p38 pathway. Cell Signal. 2013;25(1):159-67.

35. Lu LH, Lee SS, Huang HC. Epigallocatechin suppression of proliferation of vascular smooth muscle cells: correlation with c-jun and JNK. Br J Pharmacol. 1998;124(6):1227-37.

36. Saltis J, Agrotis A, Bobik A. TGF-beta 1 potentiates growth factor-stimulated proliferation of vascular smooth muscle cells in genetic hypertension. Am J Phys. 1992;263(2 Pt 1):C420-428.

37. Liu X, Sun L, Torii M, Rakic P. Connexin 43 controls the multipolar phase of neuronal migration to the cerebral cortex. Proc Natl Acad Sci U S A. 2012;109(21):8280-5
38. Slater SC, Koutsouki E, Jackson CL, Bush RC, Angelini GD, Newby AC, George SJ. R-cadherin:beta-catenin complex and its association with vascular smooth muscle cell proliferation. Arterioscler Thromb Vasc Biol. 2004;24(7):1204-10.

39. Nasim MT, Ogo T, Chowdhury HM, Zhao L, Chen CN, Rhodes C, Trembath RC. BMPR-II deficiency elicits pro-proliferative and anti-apoptotic responses through the activation of TGFbeta-TAK1-MAPK pathways in PAH. Hum Mol Genet. 2012;21(11):2548-58.

40. Mermis J, Gu H, Xue B, Li F, Tawfik O, Buch S, Bartolome S, O'Brien-Ladner A, Dhillon NK. Hypoxia-inducible factor-1 alpha/platelet derived growth factor axis in HIV-associated pulmonary vascular remodeling. Respir Res. 2011;12:103.

41. Yang S, Banerjee S, Freitas A, Cui H, Xie N, Abraham E, Liu G. miR-21 regulates chronic hypoxia-induced pulmonary vascular remodeling. Am J Physiol Lung Cell Mol Physiol. 2012;302(6):L521-529.

42. Calore M, De Windt LJ, Rampazzo A. Genetics meets epigenetics: Genetic variants that modulate noncoding RNA in cardiovascular diseases. J Mol Cell Cardiol. 2015;89(Pt A):27-34.

43. Davis BN, Hilyard AC, Nguyen PH, Lagna G, Hata A. Induction of microRNA-221 by platelet-derived growth factor signaling is critical for modulation of vascular smooth muscle phenotype. J Biol Chem. 2009;284(6):3728-38.

44. Wang $Y$, Kahaleh B. Epigenetic repression of bone morphogenetic protein receptor II expression in scleroderma. J Cell Mol Med. 2013:17(10):1291-9.

45. Zeng Y, Liu H, Kang K, Wang Z, Hui G, Zhang X, Zhong J, Peng W, Ramchandran R, Raj JU, et al. Hypoxia inducible factor-1 mediates expression of miR-322: potential role in proliferation and migration of pulmonary arterial smooth muscle cells. Sci Rep. 2015;5:12098.

46. Trapnell C, Pachter L, Salzberg SL. TopHat: discovering splice junctions with RNA-Seq. Bioinformatics. 2009;25(9):1105-11.

47. Guttman M, Garber M, Levin JZ, Donaghey J, Robinson J, Adiconis X, Fan L, Koziol MJ, Gnirke A, Nusbaum C, et al. Ab initio reconstruction of cell typespecific transcriptomes in mouse reveals the conserved multi-exonic structure of lincRNAs. Nat Biotechnol. 2010;28(5):503-10.

48. Trapnell C, Williams BA, Pertea G, Mortazavi A, Kwan G, van Baren MJ, Salzberg SL, Wold BJ, Pachter L. Transcript assembly and quantification by RNA-Seq reveals unannotated transcripts and isoform switching during cell differentiation. Nat Biotechnol. 2010;28(5):511-5.

49. Lin XC, Sui WG, Qi SW, Tang DE, Cong S, Zou GM, Zhang Y, Li H, Chen WB, Cheng $Z Q$, et al. Quantitative proteomic profiling of renal tissue in human chronic rejection biopsy samples after renal transplantation. Transplant Proc 2015;47(2):323-31.

50. Niu Y, Zhang L, Qiu H, Wu Y, Wang Z, Zai Y, Liu L, Qu J, Kang K, Gou D. An improved method for detecting circulating microRNAs with S-Poly(T) Plus real-time PCR. Sci Rep. 2015;5:15100.

\section{Submit your next manuscript to BioMed Central and we will help you at every step:}

- We accept pre-submission inquiries

- Our selector tool helps you to find the most relevant journal

- We provide round the clock customer support

- Convenient online submission

- Thorough peer review

- Inclusion in PubMed and all major indexing services

- Maximum visibility for your research

Submit your manuscript at www.biomedcentral.com/submit 